\title{
TOTAL ELECTRON CONTENT MEASUREMENTS IN THE IONOSPHERE DISTURBED BY HIGH-POWER HIGH-FREQUENCY WAVES BY THE METHODS OF INCOHERENT SCATTERING OF RADIO WAVES AND RADIO SOUNDING BY GLONASS SATELLITE SIGNALS
}

\author{
E. D. Tereshchenko, ${ }^{1}$ S. M. Cherniakov,${ }^{1}$ \\ R. Yu. Yurik, ${ }^{1 *}$ M. T. Rietveld, ${ }^{2,3}$ and I. Häggström ${ }^{2,4}$ UDC 533.9.082+533.95+537.868
}

\begin{abstract}
We present the results of comparing the total electron content measurements based on GLONASS satellite signals and the EISCAT UHF incoherent scatter radar (Tromsø, Norway) during modification of the high-latitude ionosphere in the magnetic zenith direction by high-frequency radio waves of the EISCAT/Heating facility (Tromsø, Norway). The measurements were performed during two experiment campaigns in October 2013 and in October 2018. In general, the total electron content variations obtained from the radar data in the altitude range 100-400 $\mathrm{km}$ were consistent with the total electron content variations from the GLONASS satellites. The efficiency of using GLONASS satellites for observations of high-latitude phenomena was shown. The anomalous increase in the total electron content by 4 TECU obtained from the incoherent scatter radar when the ionosphere was heated in the region close to the magnetic zenith is considered. The GLONASS satellite data show the total electron content reduction in the same region. To explain the disagreement between measurements by these two methods, the effect of smallscale electron-density irregularities arising in the region modified by high-power HF radio waves is considered. It is shown that when the electron density in artificial irregularities exceeds the background density of the medium by $2 \cdot 10^{-3}$ times in relative units, scattering by irregularities with spatial scales of the order of $16 \mathrm{~cm}$ becomes predominant in the reflected signal.
\end{abstract}

\section{INTRODUCTION}

The advent of incoherent scatter radars (ISRs) has led to the publication of a number of papers related to comparing the data of these radars with the data obtained using other measuring instruments. With the beginning of active operation of ISRs of the EISCAT European Scientific Association, it became possible to compare their data with the data obtained by other ionosphere observation techniques, including the USA Global Positioning System, GPS [1, 2], models [3], and ionosondes [4].

Early GPS users believed that incoherent scatter radars receive a large amount of reliable and fairly accurate data on the high-latitude ionosphere, which will make it possible to study the effect of various phenomena on the accuracy of determining the total electron content (TEC) by GPS satellite signals. They were especially interested in the electron density profiles obtained by ISRs along the line of sight and, therefore, the possibility to calculate TEC on their basis. ISR facilities provide TEC values on the receiversatellite line of sight, as well as on the satellite path due to moving the radar antenna patterns. The joint use of ISRs and satellite measurements makes it possible to compare the TEC obtained by these methods.

* Roman.Yurik@pgi.ru

1 Polar Geophysical Institute, Murmansk, Russia; ${ }^{2}$ European Incoherent Scatter Scientific Association EISCAT; ${ }^{3}$ Troms $\varnothing$ University - Arctic University of Norway, Norway; 4 Swedish Institute of Space Physics, Kiruna, Sweden. Translated from Izvestiya Vysshikh Uchebnykh Zavedenii, Radiofizika, Vol. 62, No. 10, pp. 747758, October 2019. Original article submitted October 25, 2019; accepted October 30, 2019. 
It was assumed that such a comparison will permit one to evaluate the possible errors in determining the TEC obtained from GPS data, as well as errors in converting TEC from oblique to vertical. One of the disadvantages of using GPS for studying processes in the high-latitude ionosphere is their low inclination $\left(55^{\circ}\right)$, which precludes considering, e. g., the processes occurring along the magnetic field lines (precipitations, etc.) more accurately.

At the end of the seventies of the last century, the USSR started work on the development and deployment of a global navigation satellite system (GNSS) of a new generation, which was the continuation of the existing satellite navigation system based on low-orbiting satellites. On this basis, a full-featured Russian global navigation satellite system GLONASS was commissioned in 2011. Currently, a constellation of GLONASS satellites comprises 28 satellites, of which 24 are working and 4 are in reserve. The GLONASS satellites have an inclination of $64.8^{\circ}$ and a flight altitude of $19100 \mathrm{~km}$, and therefore their use for observing processes in the high-latitude ionosphere is more promising.

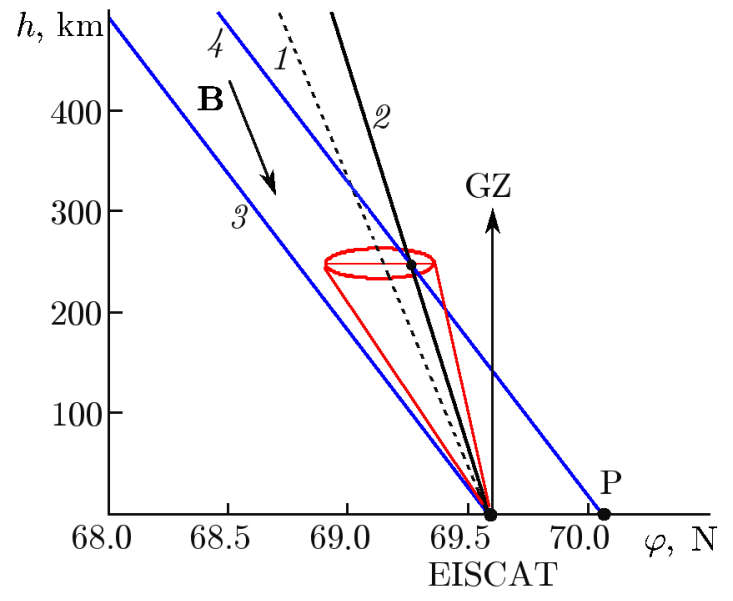

Fig. 1. Comparison of the GNSS satellite line of sight for the receivers installed at the EISCAT/Heating facility ( 1 line in the magnetic zenith direction; 2 the GLONASS satellite line; 3 the GPS satellite line) and in its vicinity ( 4 the GPS satellite line from a remote point); $h$ and $\varphi$ are the altitude and latitude, respectively.
Figure 1 shows the position of the GLONASS and GPS satellites during observation of the heating patch near the EISCAT/Heating facility $\left(69.59^{\circ} \mathrm{N}, 19.22^{\circ} \mathrm{E}\right.$, Troms $\varnothing$, Norway) [5]. Location of the heater, the ISR, and the GLONASS satellite signal receiver is denoted in the figure as EISCAT. Arrow with the letter B shows the direction of the magnetic field line in the observation region. Zenith direction at the observation site is shown by an GZ arrow. During the ionosphere heating the facility beam was magnetic-zenith directed (dashed line 1). The heating patch is shown at the center of the figure at an altitude of $250 \mathrm{~km}$ in the form of an ellipse. During heating, the height of the heating patch can be varied by tens of kilometers. It is seen that for the observer at the EISCAT site the GLONASS satellite line of sight (line 2) passes through the heating patch and the GPS satellite line of sight (line 3) lies outside the heating patch. To ensure that the same heating patch could be observed using the GPS satellite as using the GLONASS satellite, the GPS signal receiver should be moved a few tens of kilometers north of the EISCAT observation point, to point $\mathrm{P}$. The angle between the magnetic zenith direction and the satellite line of sight (line 4) increases, which leads to a worse geometry of observation of the electron density structures in the heating patch. The height of the maximum ionosphere heating and ionospheric structure variation region can be varied depending on the ionospheric and geomagnetic conditions. The GLONASS satellites make it possible to record these variations since the satellite line of sight at the reception site passes through the ionospheric variation region irrespective of the altitude at which this region is located at the moment of observation. At the same time, the GPS receiver moved a certain distance northward, e. g., to point P, to monitor the assumed heating patch, can record variations only in a limited range of altitudes. The altitude of the maximum-heating patch and conditions for the appearance of artificial ionospheric irregularities there can vary with the variation in ionospheric conditions. In this case, the TEC measured by using GPS satellites can characterize the state of the ionosphere outside or at the edge of the region of development of the main heating processes. This can give an incomplete picture of the dynamics of the ionosphere during its heating.

This geometric effect was considered in [3] in the analysis of joint observations of the ionosphere in Troms $\varnothing$ by the ISR radar and GPS satellites. When the TEC obtained by the latter were compared, it was noted that the GPS-based TEC values are sometimes less than TEC values obtained by the ISR. This mismatch has been shown to be caused by the low GPS satellite tilt. 
The use of active experiments greatly increases the possibilities of focused study of the ionosphere behavior in different conditions. The work of the heating facilities and their impact on the ionosphere by high-power HF radio waves induce artificial ionospheric irregularities (AIIs). The heater experiments established the fact of occurrence of AIIs and also identified some of their features. Therefore, the studies aimed at analyzing experimental material on AII characteristics and their dependence on various geophysical conditions are relevant to the physics of the ionosphere.

Different methods are employed to study the processes in the ionosphere during heating experiments. The first observations of the behavior of the ionospheric plasma modified by a heater were based on using an ISR located next to the EISCAT/Heating facility. It was shown that the angle between the sounding direction and the direction of the magnetic field line or position of the magnetic zenith should be taken into account in such studies for the obtained ISR data [6].

One of the most informative methods for determining the electron density variations is the use of satellite observations. First observations of the heating patch by the method of radio transmission by signals from low-orbiting navigation satellites were performed in 1998 using the EISCAT/Heating facility [7] and then at mid-latitudes using the Sura heater [8]. The appearance of GPS navigation satellites contributed to the further development of the studies of the structure of the heating patch. At mid-latitudes, observations of the ionospheric heating patch by reception of GPS signals were based on the Sura heater [9]. At high latitudes, focused studies of the processes in the heating area using GNSS satellite signals almost have not been performed to date, but paper [10], in which the heating effects at EISCAT/Heating were observed using GPS satellites, can be mentioned.

It was noted in [11] that, according to the ISR, during heating of the ionosphere by high-power HF $\mathrm{X}$-mode radio waves the EISCAT/Heating system in Troms $\varnothing$ recorded an increase in electron density $N$ over a wide range of altitudes in the magnetic zenith direction, which reached $25 \%$. To explain the increase in $N$, two mechanisms were considered in [11], namely, the ionization-recombination balance violation and stimulated ionization by accelerated electrons. Calculations by the authors of [11] showed that under conditions of the experiment, the first mechanism could give a $2.4-5.3 \%$ increase relative to the unperturbed level. Therefore, it was suggested there that in this case, at altitudes above the reflection level of high-power radio waves the generation of a flux of accelerated electrons in the field of high-power HF X-mode radio waves is the most probable mechanism for increasing the electron density $N$.

The problem of correct interpretation of the ISR data in the case of the electron density increase by tens of percent has been considered in different papers $[12,13]$. When calculating the electron density by the ISR method, in these cases one can encounter the manifestation of non-Maxwellian or other similar mechanisms of reflection, for example, reflections from satellites, meteor echoes, etc. Such effects can also be observed when the electron density is calculated in the heating experiments. Until now, this problem remains open and requires further research.

\section{OBSERVATION RESULTS}

To study the electron density variation during the ionosphere modification by high-power HF radio waves from the EISCAT/Heating facility, the heating patch was monitored using an EISCAT ultra highfrequency (UHF) incoherent scatter radar $(930 \mathrm{MHz})$ and an alternative method based on using GLONASS navigation satellite signals in two observation campaigns from October 20 to November 1, 2013.

In both cases, the GLONASS satellite signal receiving system was installed $100 \mathrm{~m}$ from the heater and $30 \mathrm{~m}$ from the ISR. In the observations, the satellite signals were recorded when the receiver system GLONASS satellite line of sight passed through the heating patch near the magnetic zenith. In both experiments, the satellite signals were recorded by a Maxor-GGDT two-frequency receiver with a sampling rate of $1 \mathrm{~Hz}$. The radar beam followed the satellite trajectory and made measurements once a minute, which were used to calculate the electron density and electron temperature profiles along the satellite line of sight. To improve the heating efficiency, the radiation pattern of the heating antenna was tilted $12^{\circ}$ southward from the vertical line towards the local magnetic zenith. Ionospheric environment in the heater region was 

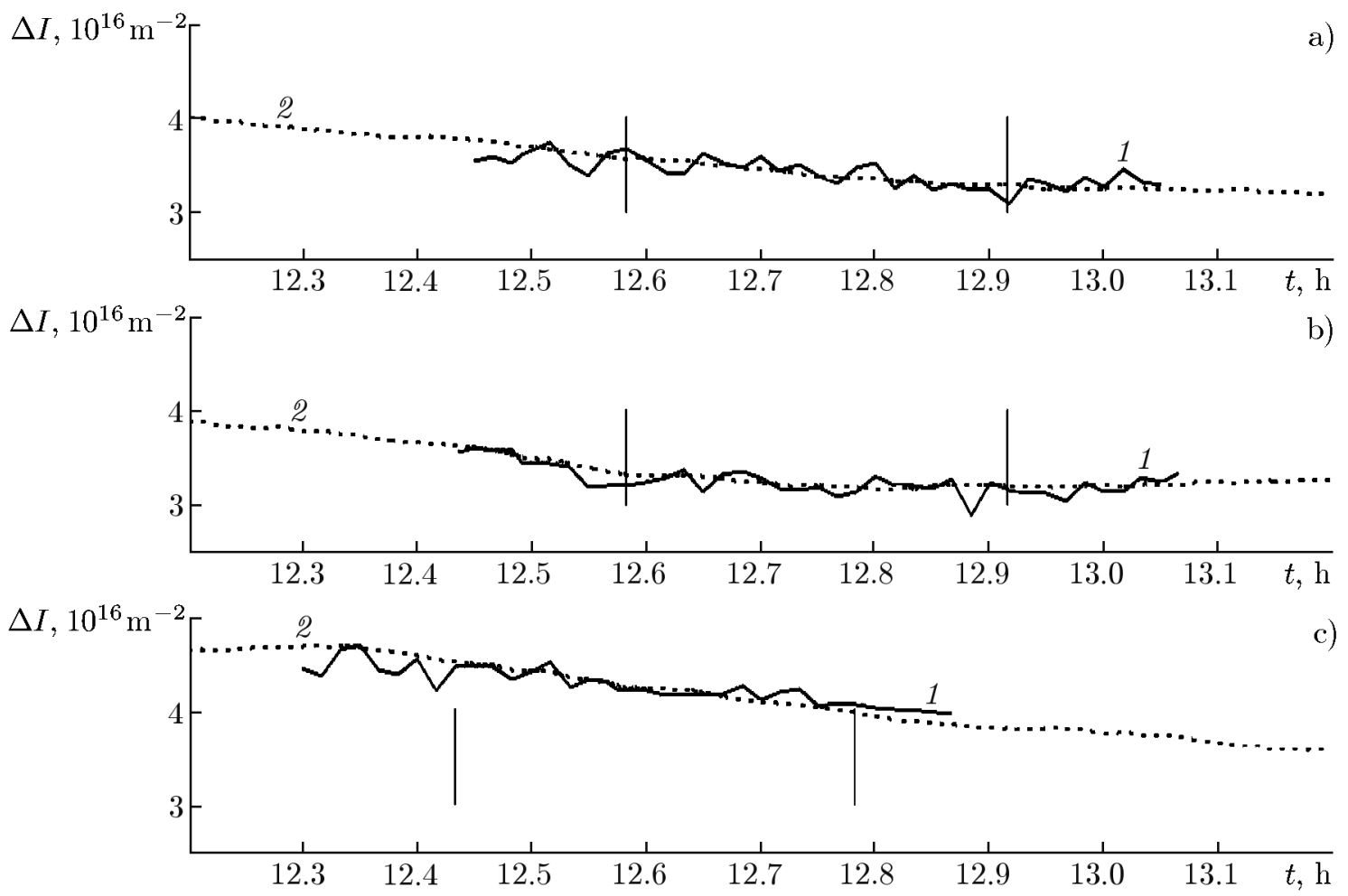

Fig. 2. The total electron content measured by the ISR (solid line 1) and the relative TEC according to the GLONASS satellites (dotted curve 2) as functions of time $t$ for measurements on October 16 (a), October 17 (b), and October 18, 2018 (c).

monitored by a dynasonde that provided vertical sounding ionograms once per $2 \mathrm{~min}$.

Between October 21 and November 1, 2013, eight sessions were held on modification of the ionosphere by high-power HF radio waves of the EISCAT/Heating facility. Signals from the GLONASS satellites and UHF ISR were simultaneously recorded in that experiment. Observations were performed in the evening, six of which were organized in quiet and two, in disturbed ionospheric and geomagnetic conditions. This paper considers the case of anomalous behavior of the electron density, which took place on October 21, 2013 during heating between 16:20 UT and 16:50 UT.

Between October 8 and October 19, 2018, five sessions were held on the ionosphere modification, when signals from the GLONASS satellites and ISR were simultaneously recorded. During the experiments of 2018, observations were carried out in the daytime (12:00-13:00 UT), two of which (October 9 and 10) took place in disturbed geomagnetic conditions. Three observations (between October 16 and October 18) were organized in quiet geomagnetic conditions. The total values of the planetary $K_{\mathrm{p}}$ index in that day were $10-, 4$, and $1-$, respectively.

Altitudes from 100 to $400 \mathrm{~km}$, for which the electron density was most reliably determined, were chosen to calculate the TEC obtained by the radar. Above $400 \mathrm{~km}$, the electron density values obtained from the radar data had a significant spread. Since below $150 \mathrm{~km}$ the TEC makes a constant contribution of no more than $5 \cdot 10^{10} \mathrm{~m}^{-2}$ [2], the TEC below $100 \mathrm{~km}$ can be neglected. In this case, the total electron content from $100 \mathrm{~km}$ to the altitudes of the satellite includes the ionospheric region controlled by the radar, as well as the ionized atmosphere at up to the satellite altitudes. In quiet conditions, the ionosphere and protonosphere areas undergo slight variations near the noon. Therefore, it can be assumed that above $400 \mathrm{~km}$ the TEC slightly changes within a half-hour observation. In this case, the TEC obtained from the satellite data can be reduced by this constant value and the TEC based on the radar data can be compared with the TEC variations based on the satellite data.

Figure 2 for October 16-18, 2018 shows the total electron content obtained by the radar for altitudes 
of 100 and $400 \mathrm{~km}$ along the receiver-satellite line of sight (solid line 1), and the TEC variations obtained from the GLONASS satellites (dashed line 2) and combined with the TEC measured by the radar. Vertical lines indicate the start and end times of heating.

The TEC variation obtained from satellite data during their one-hour flights is quite smooth for the given cases. No large-scale ionospheric features of artificial or natural origin have been detected. The behavior of the radar-based TEC is generally consistent with the TEC based on the satellite data, except for small variations due to inaccuracy in radar measurements of the electron density. Also, it follows from the diagrams that these differences in magnitude do not exceed 0.4 TECU (the generally accepted unit of total electron content $1 \mathrm{TECU}=10^{16} \mathrm{~m}^{-2}$ ).

In most cases of joint measurements of the total electron content by the radar and the satellite there was correspondence between their behaviors: the TEC variation was similar and the same undulatory behavior was observed in the TEC structure. In some cases, effective heating was absent and minor TEC variations took place.

\section{ANOMALOUS BEHAVIOR OF THE TOTAL ELECTRON CONTENT OBTAINED BY THE ISR METHOD}

Joint measurements showed that the TEC variations measured by both methods match each other within a measurement error. The results of the experiment on October 21, 2013 were the exception. We consider this case in more detail, since in that experiment there was an anomalous increase in electron density according to the ISR data, which is not consistent with the TEC measurements based on satellite radio sounding of the disturbed ionospheric region.

Geomagnetic environment on October 21, 2013 was quiet, and the total $K_{\mathrm{p}}$ index in that day was 2 . The geometry of the GLONASS R01 flight is presented in Fig. 3. Thick dotted line 1 is the projection of the satellite trajectory at an altitude of $250 \mathrm{~km}$, dotted line 2 is the heating patch at an altitude of $250 \mathrm{~km}$. Ellipses with the numbers 3,6, and 9 show the angles in degrees from

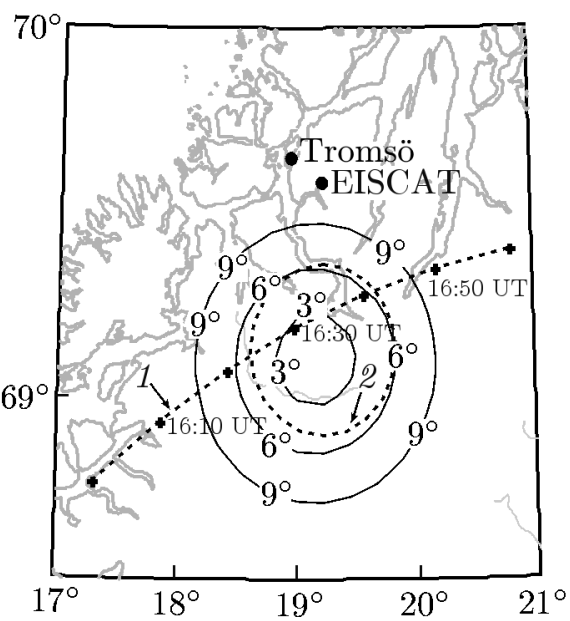

Fig. 3. Geometry of the experiment on October 21, 2013. the magnetic zenith, and EISCAT is the location of the receiver and the heater. During the flight of the satellite, the minimum angle between the receiver-satellite line of sight and the magnetic zenith was $3^{\circ}$.

In the experiment of October 21, 2013 between 16:20 UT and 16:50 UT, the ionosphere was modified by continuous radiation at a frequency of $6.2 \mathrm{MHz}$ (the cutoff frequency of the $F_{2}$ layer is $6.8 \mathrm{MHz}$ ), while the effective radiated power of the heater was about $200 \mathrm{MW}$. An antenna system providing a beamwidth of about $12.5^{\circ}$ was used in the experiments.

Figure $4 a$ shows the TEC variation (line 1) obtained from phase measurements of satellite signals on October 21, 2013 over the entire time of transit in the radio coverage area with the initial phase neglected. A black rectangle on the bottom shows the operating time of the EISCAT/Heating facility and a gray rectangle, the time interval in which the satellite line of sight passed through the heating patch. Figure $4 b$ shows the TEC variations (line 1) obtained from phase measurements of satellite signals between 15:50 UT and 17:10 UT. The trends related to the satellite motion and large-scale ionospheric variations were removed from the data. Dashed lines 2 in Figs. $4 a$ and $4 b$ show the values of the $\Xi$ angle between the satellite direction and the magnetic field (right vertical axis).

It is seen in Fig. $4 b$ that heating of the ionosphere caused a change in its structure. At the southwestern edge after the start of heating at 16:20 UT the TEC increases for $2.5 \mathrm{~min}$, then it sharply decreases by 1 TECU, and reaches a minimum at 16:27 UT. After that the TEC again increases by 0.7 TECU and 


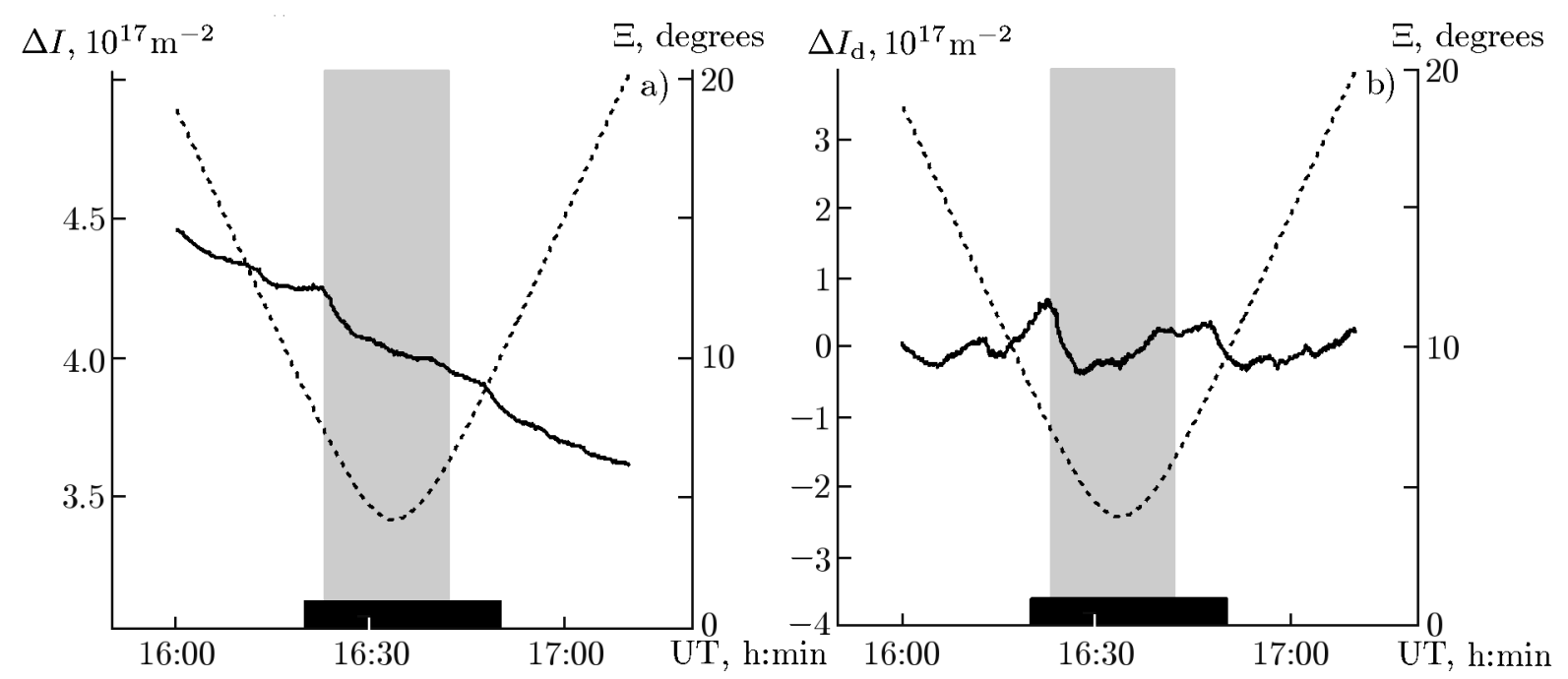

Fig. 4. The TEC variation obtained from phase measurements of satellite signals on October 21, 2013 over the entire time of transit of the satellite in the radio coverage area (a); TEC variations from 15:50 UT to 17:10 UT on October 21, $2013(b)$.

reaches a maximum at 16:40 UT at the north-eastern edge of the heating patch. Then the TEC decreases by 0.1 TECU at 16:42 UT. At this time, the satellite line of sight escapes from the heating patch, the TEC starts to increase, and the second maximum is reached at 16:47 UT. The ionosphere heating continues at this time. After that, the TEC starts to decrease sharply, although the heating continues for another 3 min. This effect is likely due to the fact that the satellite line of sight has left the area where the impact of the heating wave is manifested. It is seen in the figure that the main features of the variations in TEC are its decrease at the center of the antenna pattern, i. e., in the magnetic zenith direction, and its elevated values at the edge of the heating patch. In this case, the asymmetry of the TEC profile is observed: its minimum value is shifted south-westward with respect to the center of the antenna pattern. Similar effects during the ionosphere heating by high-power electromagnetic radiation were also previously observed [14, 15].

Figure 5 shows the change in altitude profiles of the electron density $N(h)$ based on the radar data when scanning the ionosphere in the direction of the satellite during its flight over the ionosphere heating patch. The profiles were drawn as functions of altitudes along the satellite line of sight. To obtain the electron density values we used the GUISDAP (Grand Unified Incoherent Scatter Design and Analysis Package) calculation technique $[16,17]$.

The electron density profiles (see Fig. 5) had a background character before 16:27 UT. Increased values of the electron density started to form at altitudes of 220-280 km (16:28-16:33 UT) at 16:28 UT. At first, a maximum appeared on the electron density profile at an altitude of about $270 \mathrm{~km}$ at 16:28 UT. Then at 16:29 UT the maximum electron density increased abruptly, and was a factor of three to five higher than the background electron density before 16:33 UT. At 16:34 UT, the electron density abruptly decreased to the background value.

A significant (a factor of 2.5-4.0) increase in the electron density in the region of the maximum of the $F$ layer in the central heating patch was reached in the experiment. The increasing electron density region occupies an area of about $60 \mathrm{~km}$ in altitude, between 220 and $280 \mathrm{~km}$. Thus, when the ionosphere is heated by an O-mode electromagnetic wave radiated along the magnetic zenith direction, the maximum electron-density variations occurred in a narrow altitude range near the magnetic zenith. In the period of observation, the maximum ionospheric electron density above the dynasonde was at altitudes of 230-250 km.

Figure 6 shows the TEC variations obtained on October 21, 2013 according to the GLONASS satellite (dotted line 1) and the TEC variations obtained from the ISR data for altitudes between 100 and $600 \mathrm{~km}$ (solid line 2). It is seen that the behavior of TEC variations generally coincides at the edge, but not in the central part. The total electron content obtained from the radar data shows an increase in the central 


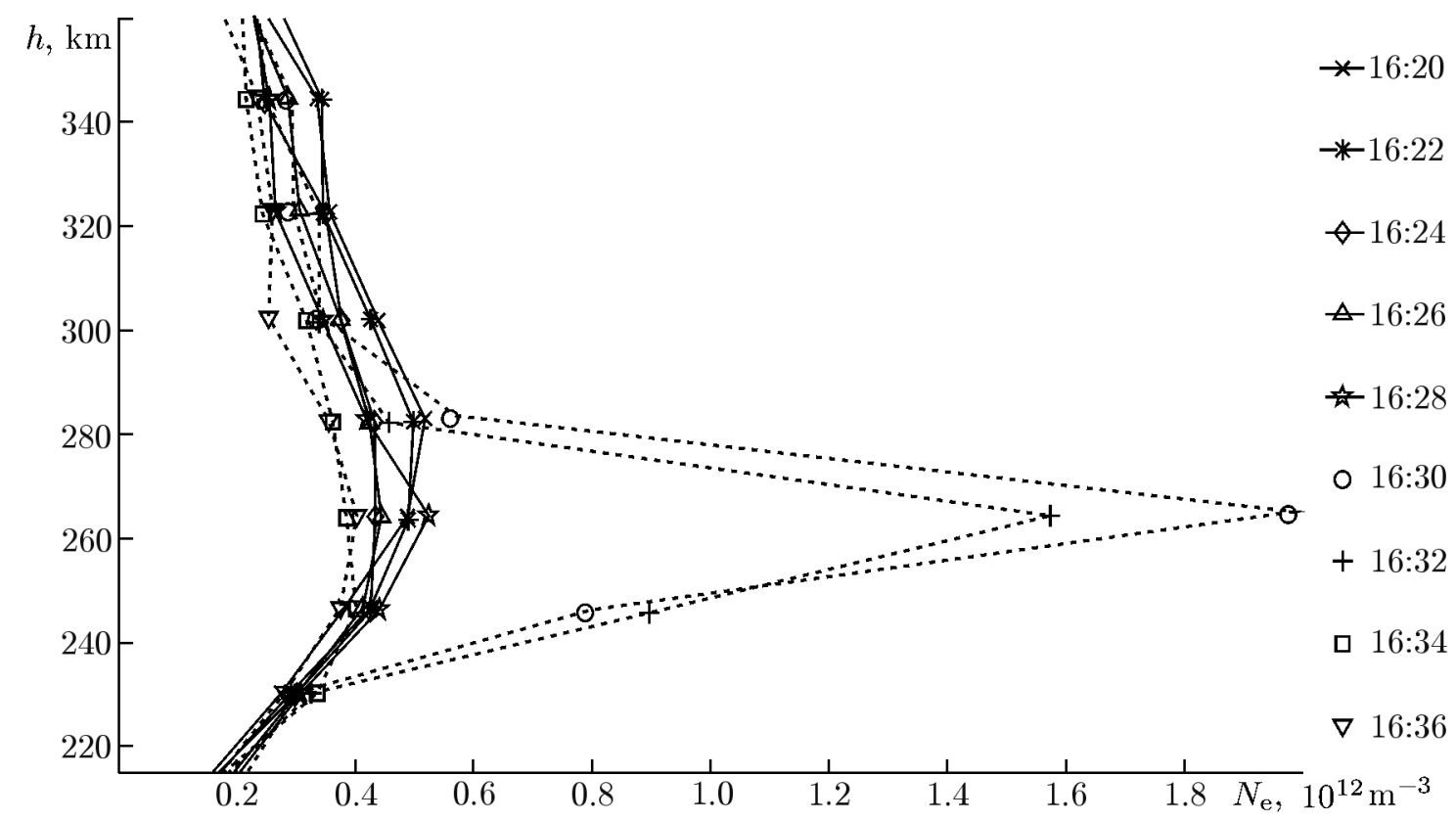

Fig. 5. Altitude profiles of the electron density $N_{\mathrm{e}}(h)$ obtained by an ISR with the ionosphere scanning in the satellite direction on October 21, 2013 at indicated instants of time.

part by 4 TECU, significantly exceeding the error in the ISR method. However, the TEC variations obtained from satellite data undergo only a slight variation.

Multiyear experience of using the ISR method indicates that in this case the anomalous increase in electron density should not be interpreted as real, since it can be due to other mechanisms. For example, the maximum electron density obtained from ISR data at an altitude of $270 \mathrm{~km}$ can be explained by the amplification of the ion line in the radar data $[18,19]$.

In our opinion, the disagreement in TEC measurements in the HF-disturbed ionosphere based on ISR and satellite data can probably be caused by the increased power of the radar signal due to backscattering of the field by the turbulized ionospheric area. We assume that the appearance of the latter is due to the effect of high-power HF waves on the ionospheric plasma.

When calculating the spectrum of an incoherently scattered signal due to the smallness of the thermal fluctuations, it is assumed that the latter do not contribute to

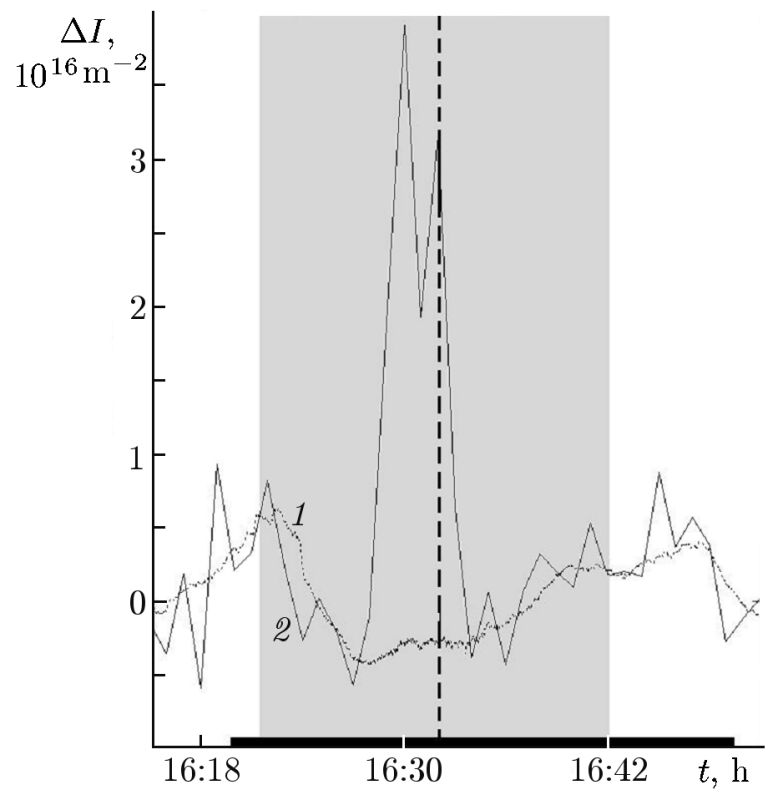

Fig. 6. Comparison of the radar-based (solid line 2) and satellite-based (dotted line 1) TEC variations on October 21, 2013. the average dielectric permittivity of the medium. However, sufficiently strong electron-density irregularities can arise in the region affected by a high-power disturbing HF field, and the single-scattering approximation cannot be used for describing the field in such a medium, since the multiply scattered fields should be taken into account. This leads to the fact that instead of the unperturbed dielectric permittivity of the medium, some effective dielectric permittivity should be used in a region with irregularities.

Let $\varepsilon$ be the dielectric permittivity of the background plasma, which is about unity in the highfrequency limit. Then for the effective dielectric permittivity $\varepsilon_{\text {eff }}$ of a plasma with irregularities when the 
field is scattered by small-scale irregularities the following formula is valid [20]:

$$
\varepsilon_{\mathrm{eff}}=1-\frac{1}{3}\left\langle\delta \varepsilon^{2}\right\rangle\left(1-2 i k^{3} a^{3}\right)
$$

Here, the angle brackets denote statistical averaging, $k=2 \pi / \lambda$ is the wave number, $a$ is a characteristic scale of irregularities,

$$
\delta \varepsilon(\mathbf{r})=-\left(4 \pi r_{e} / k^{2}\right) \delta N_{\mathrm{i}}(\mathbf{r})
$$

$r_{\mathrm{e}}=2.82 \cdot 10^{-15} \mathrm{~m}$ is the classical electron radius, $\delta N_{\mathrm{i}}(\mathbf{r})$ are the electron-density fluctuations generated in the ionosphere by high-power HF radio waves, and $\mathbf{r}$ is the radius vector. Note that Eq. (1) for $k^{3} a^{3} \ll 1$ coincides with the expression for the effective dielectric permittivity of a mixture, which is given in [21] for the static case.

Comparison of the power $S_{i}$ of an incoherently scattered signal from volume $V[22]$ and the power $S_{\mathrm{s}}$ of a signal scattered by a volume with effective dielectric permittivity $\varepsilon_{\text {eff }}[23]$ assuming that the turbulent formations are isotropic leads to the following relation:

$$
R=\frac{S_{\mathrm{i}}}{S_{\mathrm{s}}}=\left(\frac{4 \pi}{3}\right)^{2} \frac{r_{\mathrm{e}}^{2} N^{3}}{k^{4}}\left(\frac{\left\langle\delta N_{\mathrm{i}}^{2}\right\rangle}{N_{\mathrm{i}}^{2}}\right)^{2} .
$$

Consider the quantity $R$ for conditions typical of the EISCAT facility. We have $\lambda=0.32 \mathrm{~m}, N \approx$ $2 \cdot 10^{11} \mathrm{~m}^{-3}$, and $V=10^{10} \mathrm{~m}^{3}$. Substituting these values into Eq. (2), we have $R \approx 0.7 \cdot 10^{11}\left(\left\langle\delta N_{\mathrm{i}}^{2}\right\rangle / N_{\mathrm{i}}^{2}\right)^{2}$. We see that for $\sqrt{\left\langle\delta N_{\mathrm{i}}^{2}\right\rangle / N_{\mathrm{i}}^{2}}=2 \cdot 10^{-3}$ the quantity $R$ takes the value 1 . In this case, the powers of the scattered signals will be identical. Once this value is exceeded, the effect of scattering by irregularities with spatial scales $16 \mathrm{~cm}$ becomes predominant in the signal for the EISCAT UHF radar.

Phase fluctuations of the GLONASS signal can serve as some confirmation of the hypothesis about the effect of the HF-generated irregularities on the results of comparing the data obtained by radio-transmission and ISR methods.

For simplicity and convenience of the analysis, consider the difference $F$ between the reduced pseudo ranges of the GLONASS signals measured at the frequencies $f_{1}$ and $f_{2}$ :

$$
F=L_{1} \lambda_{1}-L_{2} \lambda_{2}
$$

where $L_{1}$ and $L_{2}$ are the carrier-frequency phases measured in integer cycles at frequencies $f_{1}$ and $f_{2}$, respectively, and $\lambda_{1}$ and $\lambda_{2}$ are the wavelengths corresponding to these frequencies. For the GLONASS satellites, the frequencies of coherent signals are determined as

$$
f_{1}=1602+(9 / 16) k \mathrm{MHz}, \quad f_{2}=1246+(7 / 16) k \mathrm{MHz},
$$

where $k$ is the number of the channel.

As the signals pass through the ionosphere, the parameter $F$ includes two components:

$$
F=F_{0}+\Delta F
$$

The component $F_{0}$ is related to the uniform ionosphere and is proportional to the integral electron content along the satellite-receiver line, which gradually varies with the electron density variation, whereas the quantity $\Delta F$ is a fluctuation component stipulated by the non-uniformity of the ionosphere. For its analysis, the low-frequency component below the cutoff frequency $f_{\mathrm{c}}=0.35 \mathrm{~Hz}$ was removed from $F$.

Figure 7 shows the $\Delta F$ fluctuations (line 1) on October 21, 2013 during the ionosphere heating between 16:20 UT and 16:50 UT. The heating time in the figure is indicated by a black rectangle, the gray area in the center is the time of intersection of the receiver-satellite line and the antenna array pattern of the heater at a power level of $-3 \mathrm{~dB}$. Curve 2 in the figure displays the angle $\Xi$ between the receiver-satellite 


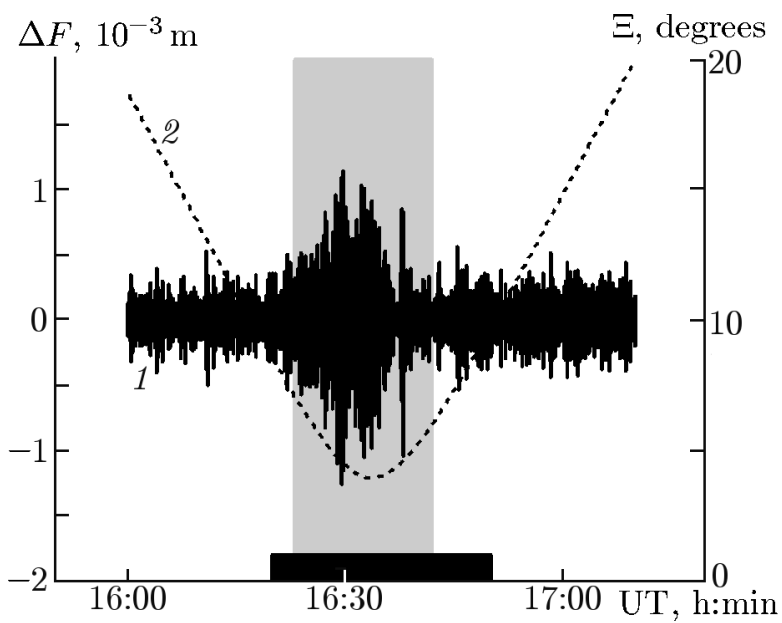

Fig. 7. The $\Delta F$ fluctuations between $16: 00$ and 17:10 UT on October 21, 2013.

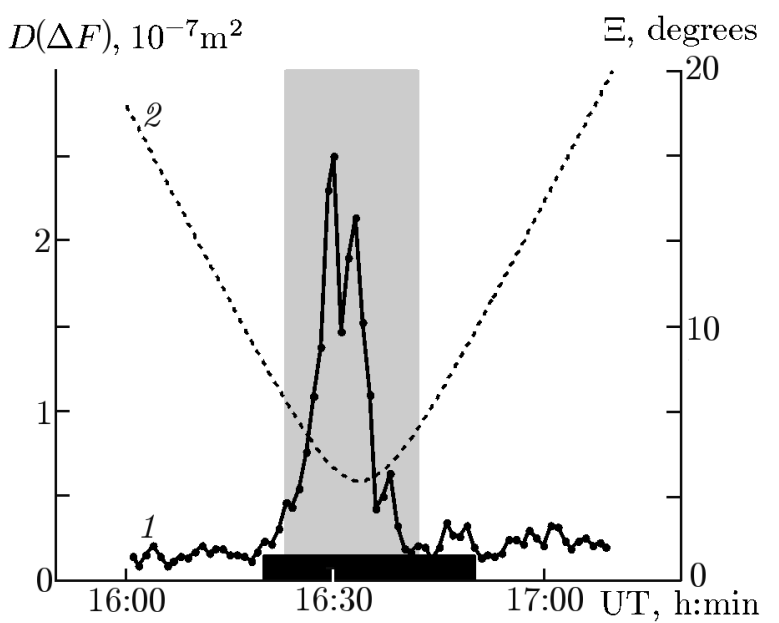

Fig. 8. Spatiotemporal variance of the $\Delta F$ fluctuations.

line and the Earth's magnetic field at an altitude of $250 \mathrm{~km}$.

It is seen in Fig. 7 that when radio signals pass through the disturbed ionospheric region, the $\Delta F$ fluctuations increase with decreasing angle between the receiver-satellite line and the magnetic field, reaching a factor of 3-4 greater values than outside the disturbed area. The observed increase in fluctuations can be explained by the presence of small-scale irregularities in the disturbed ionospheric region and small angles of the probing radio beam with a magnetic field, leading to increased flicker of the satellite signal.

Figure 8 shows the variance of $\Delta F$ fluctuations (line 1) for the same experiment. The variance is calculated at $120 \mathrm{~s}$ intervals with a $60 \mathrm{~s}$ step over the entire range of measurements. The auxiliary lines in Fig. 8 are the same as in Fig. 7 . It can be seen in the figure that the variance of the $\Delta F$ fluctuations is significantly increased in the disturbed ionospheric region. It should be noted that the observed spatiotemporal variation of the variance of $\Delta F$ fluctuations repeats the variation of the integral electron content according to the ISR (see Fig. 6).

\section{CONCLUSIONS}

It is shown that variations of the total electron content obtained from the incoherent scatter radar and GLONASS satellites basically match each other.

Observations by an incoherent scatter radar on October 21, 2013 during the ionosphere modification by high-power HF radio waves indicated the case of abrupt increase in the electron density obtained in the magnetic zenith direction using standard calculation. The electron density profiles during scanning of the heating region corresponded to the background values, except for the region near the magnetic zenith, where there was an abrupt increase in electron density. Calculation of the total electron content on the radar-satellite line obtained from the radar along the satellite trajectory also showed a sharp increase in the total electron content near the magnetic zenith.

At the same time, according to the GLONASS satellite observations, the TEC increase was not observed. The main features of the behavior of the total electron content during continuous heating of the ionosphere for $30 \mathrm{~min}$ in the magnetic zenith direction according to the GLONASS satellite were a decrease in total electron content in the central region of the antenna pattern, i. e., in the magnetic zenith direction, and elevated TEC values at the edge of the heating patch.

The TEC behavior according to the GLONASS satellite and the TEC behavior according to the incoherent scatter radar were largely consistent with each other, except for the central part. This difference can be explained by the fact that the coherent component in the received-signal power, which is stipulated by the electron density irregularities in the heating patch where the electron density is determined, affects the calculation results. 
It has been shown that at high latitudes in the EISCAT/Heating experiments, the GLONASS satellites, the orbit parameters of which permit one to study the TEC variations along the geomagnetic field line at the observation site, can conveniently be used to examine the ionosphere structure in the region of the ionosphere modification by a high-power $\mathrm{HF}$ radio wave.

We are grateful to the EISCAT Scientific Association for the allocation of working hours of the EISCAT/Heating facility and UHF incoherent scatter radar as part of the EISCAT Peer-reviewed Program experiments and for the possibility of GNSS signal observation at the EISCAT Observatory (Troms $\varnothing$ ).

\section{REFERENCES}

1. B. Forte, N. D. Smith, C. N. Mitchell, et al., Ann. Geophys., 31, 745 (2013).

2. N. Jakowski, E. Sardon, E. Engler, et al., Ann. Geophys., 14, 1429 (1996).

3. J. Lilensten and Lj. R. Cander, J. Atmos. Solar-Terr. Phys., 65, 833 (2003).

4. J. Lilensten, Lj. R. Cander, M. T. Rietveld, et al., Ann. Geophys., 23, 183 (2005).

5. M. T. Rietveld, H. Kohl, H. Kopka, et al., J. Atmos. Terr. Phys., 55, 577 (1993).

6. M. T. Rietveld, M.J. Kosch, N.F. Blagoveshchenskaya, et al., J. Geophys. Res., 108, No. A4, 1141 (2003).

7. E. D. Tereshchenko, B. Z. Khudukon, M. T. Rietveld, et al., Ann. Geophys., 16, No. 7, 812 (1998).

8. E. D. Tereshchenko, B. Z. Khudukon, A. V. Gurevich, et al., Phys. Lett. A, 325, Nos. 5-6, 381 (2004).

9. E. D. Tereshchenko, A. N. Milichenko, V.L. Frolov, et al., Radiophys. Quantum Electron., 51, No. 11, 842 (2008).

10. V.E. Kunitsyn, A. M. Padokhin, E. S. Andreeva, et al., in: Proc. of the XXXth URSI General Assembly and Scientific Symposium, 13-20 August 2011, Istanbul, Turkey, Art. No. 6051133.

11. N.F. Blagoveshchenskaya, T.D. Borisova, T.K. Yeoman, et al., Radiophys. Quantum Electron., 53, Nos. 9-10, 512 (2010).

12. M.Lockwood, K. Suvanto, J.-P. Maurice, et al., J. Atmos. Terr. Phys., 50, Nos. 4-5, 467 (1988).

13. J. Wu, J. Wu, M. T. Rietveld, et al., J. Geophys. Res.: Space Phys., 122, 1277 (2017).

14. V.L. Frolov, Radiophys. Quantum Electron., 55, 110 (2012).

15. V.E. Kunitsyn, E. S. Andreeva, V. L. Frolov, et al., Radio Sci., 47, RS0L15 (2012).

16. M.S.Lehtinen and A.Huuskonen, J. Atmos. Terr. Phys., 58, 435 (1996).

17. M.S.Lehtinen, A.Huuskonen, and J.Pirttila, Ann. Geophys. Atmos. Hydrospheres Space Sci., 14, No. 12, 1487 (1996).

18. T. Rexer, B. Gustavsson, T. Leyser, et al., SGO Report No. 68, 94 (2019).

19. M. T. Rietveld and A. Senior, SGO Report No. 68, 95 (2019).

20. F. G. Bass, S. Ya. Braude, E. A. Kaner, and A. V. Men', Sov. Phys. Usp., 4, No. 1, 51 (1961).

21. L. D. Landau and E. M. Lifshitz, Course of Theoretical Physics, Vol. 8, Electrodynamics of Continuous Media, Pergamon Press, Oxford, UK (1960).

22. V.E. Kunitsyn, E. D. Tereshchenko, and E.S. Andreeva, Radio Tomography of the Ionosphere [in Russian], Fizmatlit, Moscow (2007).

23. The Collected Papers of Albert Einstein, Volume 3 [Russian translation], Nauka, Moscow (1966), p. 216. 